\title{
Late presentation of upper airway obstruction in Pierre Robin sequence
}

\author{
A C Wilson, D J Moore, M H Moore, A J Martin, R E M Staugas, J D Kennedy
}

\begin{abstract}
A retrospective review was carried out of 11 consecutive patients with the Pierre Robin sequence referred to a tertiary paediatric referral centre over a five year period from 1993 to 1998 . Ten patients were diagnosed with significant upper airway obstruction; seven of these presented late at between 24 and 51 days of age. Failure to thrive occured in six of these seven infants at the time of presentation, and was a strong indicator of the severity of upper airway obstruction. Growth normalised on treatment of the upper airway obstruction with nasopharyngeal tube placement. All children had been reviewed by either an experienced general paediatrician or a neonatologist in the first week of life, suggesting that clinical signs alone are insufficent to alert the physician to the degree of upper airway obstruction or that obstruction developed gradually after discharge home. The use of polysomnography greatly improved the diagnostic accuracy in assesssing the severity of upper airway obstruction and monitoring the response to treatment. This report highlights the prevalence of late presentation of upper airway obstruction in the Pierre Robin sequence and emphasises the need for close prospective respiratory monitoring in this condition. Objective measures such as polysomnography should be used, as clinical signs alone may be an inadequate guide to the degree of upper airway obstruction. (Arch Dis Child 2000;83:435-438)
\end{abstract}

Keywords: Pierre Robin sequence; upper airway obstruction; failure to thrive

The triad of micrognathia, $U$ shaped cleft palate, and glossoptosis commonly complicated by upper airway obstruction, was originally described by a French stomatologist, Pierre Robin in the 1920s. ${ }^{12}$ Infants with Pierre Robin sequence are classically described as developing airway obstruction soon after birth. ${ }^{3}$ However, this view was challenged by Ogborn and Pemberton, ${ }^{4}$ who reported that five of $16(30 \%)$ of their cases presented with upper airway obstruction between 2 and 21 days of age. Subsequently Bull et al noted that upper airway obstruction may appear over the first month of life, but did not provide any specific clinical details. ${ }^{5}$ Late onset of upper airway obstruction, particularly after initial hospital discharge, has potentially serious consequences. Indeed deaths caused by sudden deterioration in the degree of upper airway obstruction in the Pierre Robin sequence have been reported by Caouette-Laberge et al. ${ }^{6}$ This report highlights the hitherto unreported high prevalence of late clinical presentation of upper airway obstruction in the Pierre Robin sequence, and the need for close prospective respiratory monitoring of all such infants.

\section{Materials and methods}

A retrospective case note analysis was carried out for all infants referred to, or born at, the Women's and Children's Hospital (WCH) in Adelaide with a diagnosis of Pierre Robin sequence, between February 1993 and February 1998 . This is the only tertiary referral paediatric hospital in the state of South Australia. Eleven infants, who were referred to or born at the WCH over this five year period, were diagnosed as having Pierre Robin sequence; 10 of these infants were diagnosed with upper airway obstruction. Of these 10, only two had clinically apparent obstruction on day 1 . One infant was referred from another hospital at 24 days of age with a nasopharyngeal tube (NPT), which had been in situ for one week. The remaining seven infants were not felt, by experienced paediatricians or neonatologists, to have airway obstruction during the initial neonatal period. These seven represented or were referred at 24-51 days of age (mean 36.6 days), and were subsequently diagnosed with significant upper airway obstruction. The case histories of the seven "late" presenters are reported below.

\section{Case reports}

CASE

This female infant was born at term at another metropolitan hospital. Her micrognathia and cleft palate were immediately apparent but there was no clinical evidence of upper airway obstruction. Feeding was commenced via a squeeze bottle, with no initial problems. Prior to discharge on day 7 she was reviewed by an experienced general paediatrician, who felt that she was well and without upper airway obstruction. On day 25 she was reviewed again by her general paediatrician; her weight gain was poor and her parents were concerned by her intermittent noisy breathing. She was referred to the WCH for further assessment.

Initially upper airway obstruction was not clinically obvious and she was managed with nasogastric feeds. As she did not gain weight, she was referred for respiratory evaluation and a polysomnogram was performed. This showed significant upper airway obstruction with frequent oxygen desaturation into the $80 \mathrm{~s}$. An 
NPT was placed, and the nasogastric feeds were continued. Weight gain improved and the NPT was successfully removed on day 102 . A subsequent polysomnogram showed only mild upper airway obstruction without oxygen desaturation below $94 \%$.

CASE 2

This male infant was born by caesarean section at term at another metropolitan hospital. Micrognathia and cleft palate were apparent at birth, but there was no clinically obvious upper airway obstruction. There were problems in establishing feeding and his weight gain was poor. He was managed on bolus nasogastric feeds but still failed to thrive. He was referred to the WCH on day 46 for further assessment of his weight gain. A polysomnogram performed soon after admission showed significant upper airway obstruction, with oxygen desaturation into the 80s. An NPT was placed and a follow up polysomnogram showed only mild obstruction with no significant oxygen desaturation. The NPT was removed after a further 62 days. A subsequent polysomnogram showed significant ongoing upper airway obstruction. Nasal mask continuous positive airway pressure (CPAP) was commenced for an additional three months. A further polysomnogram was normal after CPAP had been discontinued.

CASE 3

This male infant was born at 38 weeks gestation at a nearby country hospital by caesarean section. His cleft palate and micrognathia were noted, but there was no clinical evidence of upper airway obstruction when he was seen by his general paediatrician. $\mathrm{He}$ was referred to the $\mathrm{WCH}$ on day 33 with poor weight gain and noisy breathing during feeding. Upper airway obstruction was suspected and an overnight oximetry was performed showing frequent oxygen desaturation into the 80 s. An NPT was placed and nasogastric feeds were commenced. His weight gain improved and the NPT was removed after 28 days. A subsequent polysomnogram showed mild upper airway obstruction, without significant oxygen desaturation.

CASE 4

This male infant was born at a teaching hospital at 35 weeks gestation by caesarean section. He developed moderately severe hyaline membrane disease on day 1 and required intubation and intermittent positive pressure ventilation. His micrognathia and cleft palate were noted soon after birth. He was successfully extubated on day 5 and was not felt to have any upper airway obstruction by a neonatologist. On day 14 he was transferred to the WCH for surgical management of his congenital talipes equinovarus. Again no upper airway obstruction was noted by his neonatologist. He had some difficulty in establishing feeds, and had a persistent oxygen requirement, which was thought to be secondary to evolving chronic lung disease.

On day 32, after a feed, he became apnoeic and bradycardic and briefly required external cardiac massage and bag and mask intermittent positive pressure ventilation. $\mathrm{He}$ was transferred to the paediatric intensive care unit for further management. His breathing pattern on arrival was noted to be significantly obstructed and his arterial $\mathrm{pCO}_{2}$ was increased at $72 \mathrm{~mm} \mathrm{Hg}$ (by this time several hours after the acute event). An NPT was placed, his obstructive breathing improved, and his arterial $\mathrm{pCO}_{2}$ normalised over subsequent days. Following NPT removal a polysomnogram showed significant ongoing obstruction. $\mathrm{He}$ was treated with nasal CPAP with increasing pressures; however, follow up polysomnograms showed continuing severe upper airway obstruction with notable oxygen desaturation to the low $80 \mathrm{~s}$. He was commenced on nocturnal bilevel positive airway pressure via a nasal mask, and continues to require this support at 30 months of age.

\section{CASE 5}

This male infant, the second of twins, was born at the WCH by caesarean section at 37 weeks gestation. His cleft palate and micrognathia were noted soon after birth. During his initial 10 day hospitalisation he was seen regularly by a neonatologist who found no evidence of upper airway obstruction. He returned to the WCH at 24 days of age with "rapid noisy breathing" and poor weight gain, raising the suspicion of upper airway obstruction. An overnight oximetry trace showed frequent oxygen desaturation into the mid 80s and capillary $\mathrm{pCO}_{2}$ was increased. An NPT was placed and nasogastric feeds were commenced. His NPT remained in situ for 60 days, during which he had good weight gain. Polysomnograms after NPT removal have shown only mild residual upper airway obstruction.

CASE 6

This male infant was born at the WCH by normal vaginal delivery at 35 weeks gestation. Cleft palate and micrognathia were noted on day 1 but there was no clinical evidence of upper airway obstruction during his 19 day hospitalisation. He re-presented to the WCH on day 51 with failure to thrive and respiratory distress. A continuous overnight oximetry tracing was notably abnormal with frequent oxygen desaturation to the mid 80s. Capillary $\mathrm{pCO}_{2}$ was increased. An NPT was placed and nasogastric feeds were commenced. A repeat overnight oximetry was normal in air with the NPT in situ. His weight gain improved and the NPT was removed after 60 days. A follow up polysomnogram showed no significant obstruction.

CASE 7

This male infant was born by normal vaginal delivery at term in the WCH. Apart from the obvious cleft palate and micrognathia his neonatal course was uncomplicated; he was not felt by his neonatologist to have upper airway obstruction, and he was discharged on day 5 . $\mathrm{He}$ re-presented to the $\mathrm{WCH}$ on day 45 with poor weight gain and respiratory distress during feeds. Capillary $\mathrm{pCO}_{2}$ was mildly increased and he frequently became desaturated 
on the ward. He was managed conservatively with supplemental oxygen and increased energy feeds. Although upper airway obstruction was at times clinically apparent, NPT placement was not felt to be required. A recent polysomnogram at 5 months of age was normal.

\section{Discussion}

This series highlights the potential for late presentation of upper airway obstruction in the Pierre Robin sequence. Of 10 patients with upper airway obstruction, seven (70\%) presented between days 24 and 51 . All infants had been seen by an experienced neonatologist or general paediatrician, and were not thought to have clinically significant upper airway obstruction. These results suggest that either significant upper airway obstruction is not being detected clinically by experienced neonatal or paediatric staff, or that obstruction is developing gradually in these infants.

While most reported series of infants with Pierre Robin sequence have not specifically stated the age at presentation of airway obstruction, the classical view has been that, if present, airway obstruction will be apparent from birth. This view is supported by the series of Benjamin and Walker. ${ }^{7}$ In this series 24 of 26 patients with Pierre Robin sequence had symptoms of obstruction apparent before 12 hours of age. The largest series of patients with late presentation of upper airway obstruction is that of Ogborn and Pemberton, who reported five infants presenting between the third and twenty first postnatal day. ${ }^{4}$ These five patients were outnumbered by the 11 who presented on the first day of life. Bull et al have noted that significant obstruction can appear over the first one to four weeks of life. ${ }^{5}$ Furthermore, Gilhooly et al have reported two patients presenting with apparent life threatening events at about 2 months of age as their first evidence of upper airway obstruction. ${ }^{8}$ Again the vast majority of patients in this series (13 of 15), had clinically apparent airway obstruction on day 1 .

In contrast to the series listed above the majority infants in this series (seven of 10), were late presenters with upper airway obstruction. In addition the age range at presentation, from day 24 to day 51 , is outside the range previously reported by Ogborn and. Pemberton. ${ }^{4}$ As all patients had been previously assessed by either an experienced general paediatrician or a specialist neonatologist, it is possible that upper airway obstruction developed progressively in these infants. There is polysomnographic evidence that this can occur. Gilhooly et al reported that one infant had a normal initial polysomnogram but subsequently an abnormal study at 13 days of age with significant upper airway obstruction. ${ }^{8}$ In support of this, Smith also reported that respiratory obstruction in Pierre Robin sequence may be present at birth or may become progressively worse at 6 to 8 weeks of age. ${ }^{9}$ These findings suggest that the absence of clinical upper airway obstruction in the early neonatal period may not guarantee that obstruction will not develop.
Two of the patients in this series were premature; both were born at 35 weeks gestation. One of these infants developed very severe upper airway obstruction at approximately 40 weeks gestational age. The other infant developed obstruction at two weeks corrected age. It has been previously reported that premature infants tend not to develop obstruction until nearly term gestational age. ${ }^{5}$ Furthermore, in a large series of 125 patients with Pierre Robin sequence there was a $60 \%$ mortality for premature infants as opposed to a $9.6 \%$ mortality for term babies. ${ }^{6}$ Although the high mortality in this study may be in part a result of the analysis extending back to 1964 , it would still suggest that premature infants need to be closely monitored for the development of late upper airway obstruction.

Six of the seven infants in this series were assessed to have had inadequate weight gain at the time of their presentation or referral. Shprintzen has pointed out that failure to thrive in Pierre Robin sequence is almost always related to upper airway obstruction, rather than feeding problems related to the cleft. ${ }^{10}$ In a retrospective survey of 40 infants with Pierre Robin sequence, Heaf et al found that failure to thrive was significantly correlated to the degree of airway obstruction. ${ }^{11}$ These investigators were able to show a substantial decrease in the work of breathing in three infants who had their airway obstruction relieved by NPT placement. This series provides further evidence that failure to thrive in the Pierre Robin sequence should be considered as an indicator of upper airway obstruction, and that infants who are failing to gain weight need to be promptly assessed for this.

This series of patients illustrates the need for close prospective monitoring of all infants born with Pierre Robin sequence. Clinical grounds alone are clearly not sufficient to predict whether an infant will subsequently present with upper airway obstruction, suggesting that there is a role for polysomnography as a diagnostic tool in Pierre Robin sequence. The value of polysomnography in evaluating upper airway obstruction in patients with Pierre Robin sequence has been highlighted by several investigators. ${ }^{581213}$ Despite this a recent survey of paediatric otolaryngology teaching programmes in the United States and Canada found a wide variation in the use of polysomnography in this condition. ${ }^{14}$ Only nine of 23 centres surveyed thought that polysomnography was indicated for most patients with Pierre Robin sequence, and just one of these centres routinely carried out this investigation on all patients before hospital discharge. There was also considerable disagreement about whether single or serial studies were required. Serial polysomnography may be necessary in view of the findings of Gilhooly et $a l .{ }^{8}$ The notable phenotypic and pathogenetic variability of Pierre Robin sequence, which has recently been highlighted by Cohen, ${ }^{15}$ provides further support for the use of objective diagnostic techniques to detect upper airway obstruction. Where polysomnography cannot 
be easily performed, there may be a role for continuous overnight pulse oximetry. All infants with Pierre Robin sequence clearly need close clinical monitoring with particular attention paid to weight gain. It would be reasonable to suggest that all such infants who do not thrive should have a polysomnogram performed even if they have previously had a normal overnight oximetry.

A recent study by Vegter et al has shown that infants with Pierre Robin sequence do not undergo mandibular "catch up" growth. ${ }^{16}$ If this "catch up" growth does not occur, then infants with the Pierre Robin sequence may continue to have problems with upper airway obstruction as they mature. Two of our patients had polysomnographic evidence of significant airway obstruction after NPT removal. One infant continues to require nocturnal bilevel positive airway pressure at 30 months of age for severe obstructive sleep apnoea (case 4 ). There have also been a number of reports of older children with Pierre Robin sequence presenting with severe obstructive sleep apnoea. ${ }^{17}{ }^{18}$ Older children with Pierre Robin sequence have also been shown to have persistent minor abnormalities on polysomnogram. ${ }^{19}$ This suggests that children with Pierre Robin sequence need to be closely clinically monitored for the persistence or reappearance of obstructive sleep apnoea, and that further polysomnography may be required. This clinical monitoring may need to continue into adult life.

In summary, this series draws attention to the high prevalence of late clinical presentation of airway obstruction in the Pierre Robin sequence, and the need for close prospective respiratory monitoring, where possible by serial polysomnography, of these infants. Particular attention needs to be paid to premature infants who characteristically develop obstruction at around term gestational age. The pres- ence of failure to thrive in infants with Pierre Robin sequence should be assumed to be caused by airway obstruction unless proven otherwise.

1 Robin P. La chute de la base de la langue considérée comme une nouvelle cause de gêne dans la respiration nasopharyngienne. Bull Acad Med Paris 1923;89:37-41.

2 Robin P. Glossoptosis due to atresia and hypotrophy of the mandible. Am $\mathcal{F}$ Dis Child 1934:48;541-7.

3 Dennison WM. The Pierre Robin syndrome. Pediatrics 1965;36:336-41.

4 Ogborn MR, Pemberton PJ. Late development of airway obstruction in the Robin anomolad (Pierre Robin syndrome) in the newborn. Aust Paediatr f 1985;21:199-200.

5 Bull MJ, Givan DC, Sadove AM, Bixler D, Hearn D. Improved outcome in Pierre Robin sequence: effect of
multidisciplinary evaluation and management. Pediatrics 1990;86:294-301.

6 Caouette-Laberge L, Bayet B, Larocque Y. The Pierre Robin sequence: review of 125 cases and evolution of treatment modalities. Plast Reconstr Surg 1994;93:934-42.

7 Benjamin B, Walker P. Management of airway obstruction in the Pierre Robin sequence. Int $\mathcal{F}$ Pediatr Otorhinolaryngol 1991;22:29-37.

8 Gilhooly JT, Smith JD, Howell LL, Deschaine BL, Richey SL. Bedside polysomnography as an adjunct in the management of infants with Robin sequence. Plast Reconstr Surg 1993;92:23-7.

9 Smith JD. Treatment of airway obstruction in Pierre Robin syndrome. Arch Otolayngol 1981;107:419-21.

10 Shprintzen RJ. The implications of the diagnosis of Robin sequence. Cleft Palate Craniofac F 1992;29:205-9.

11 Heaf DP, Helms PJ, Dinwiddle R, Matthew DJ. Nasopharyngeal airways in Pierre Robin syndrome. $\mathcal{F}$ Pediatr pharyngeal airways

12 Freed G, Pealman MA, Brown AS, Barot LR. Polysomnographic indications for surgical intervention in Pierre Robin sequence: acute airway management and follow-up studies after repair and take-down of tongue-lip adhesion. Cleft Palate F 1988;25:151-5

13 Tomaski SM, Zalzal GH, Saal HM. Airway obstruction in the Pierre Robin sequence. Laryngoscope 1995;105:111-14.

14 Myer III CM, Reed JM, Cotton RT, Willging JP, Shott SR. Airway management in Pierre Robin sequence. Otolaryngol Head Neck Surg 1998;118:630-5.

15 Cohen MM Jr. Robin sequences and complexes: causal heterogeneity and pathogenetic/phenotypic variability. $\mathrm{Am}$ 7 Med Genet 1999;84:311-15.

16 Vegter F, Hegter JJ, Mulder JW. Pierre Robin syndrome: mandibular growth during the first year of life. Ann Plast Surg 1999;42:154-7.

17 Reimao R, Papaiz EG, Papaiz LF. Pierre Robin sequence and obstructive sleep apnea. Arq Neuropsiquiatr 1994;52: and ob $554-9$.

18 Deegan PC, Mcglone B, McNicholas WT. Treatment of Robin sequence with nasal CPAP. F Laryngol Otol 1995;109:328-30.

19 Spier S, Rivlin J, Rowe RD, Egan T. Sleep in Pierre Robin sequence. Chest 1986;90:711-15. 